\title{
Fourth universal definition of myocardial infarction. Selected messages from the European Society of Cardiology document and lessons learned from the new guidelines on ST-segment elevation myocardial infarction and non-ST-segment elevation-acute coronary syndrome
}

Justyna Domienik-Karłowicz ${ }^{1,2 *}$, Karolina Kupczyńska ${ }^{1,3 *}$, Błażej Michalski ${ }^{1,3}$, Agnieszka Kapłon-Cieślicka, ${ }^{1,4}$, Szymon Darocha, ${ }^{1,5}$, Piotr Dobrowolski ${ }^{1,6}$, Maciej Wybraniec ${ }^{1,7}$, Wojciech Wańha ${ }^{1,8}$, Miłosz J. Jaguszewski ${ }^{1,9}$

${ }^{1}$ Club 30, Polish Cardiac Society, Poland

${ }^{2}$ Department of Internal Medicine and Cardiology with the Center for Diagnosis and

Treatment of Venous Thromboembolism, Medical University of Warsaw, Poland

${ }^{3}$ Department of Cardiology, W. Bieganski Hospital, Medical University of Lodz, Poland

${ }^{4}$ Chair and Department of Cardiology, Medical University of Warsaw, Poland

${ }^{5}$ Department of Pulmonary Circulation, Thromboembolic Diseases and Cardiology, Center of Postgraduate Medical Education, European Health Center, Otwock, Poland

${ }^{6}$ Department of Hypertension, National Institute of Cardiology, Warsaw, Poland

${ }^{7}$ First Department of Cardiology, School of Medicine in Katowice,

Medical University of Silesia, Katowice, Poland

${ }^{8}$ Department of Cardiology and Structural Heart Diseases, Medical University of Silesia, Katowice, Poland

${ }^{9}$ First Department of Cardiology, Medical University of Gdansk, Poland

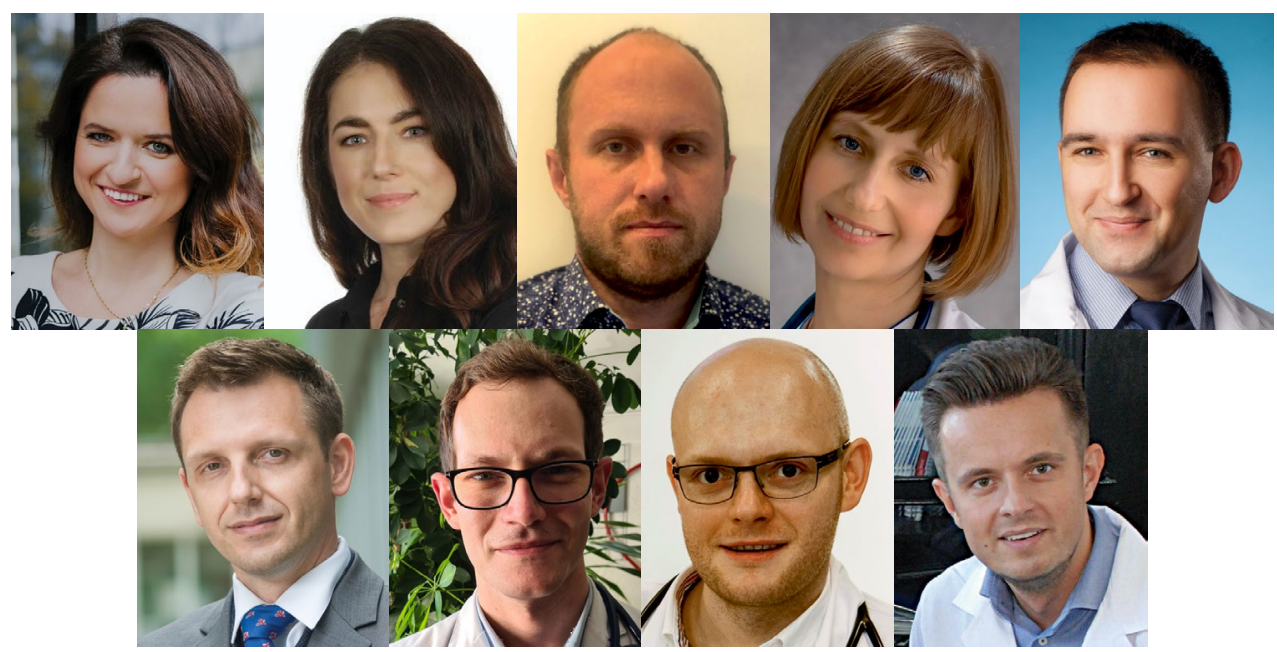

Address for correspondence: Justyna Domienik-Karłowicz, MD, PhD, Department of Internal Medicine and Cardiology with the Center for Diagnosis and Treatment of Venous Thromboembolism, Medical University of Warsaw, ul. Lindleya 4, 00-005 Warszawa, Poland, tel: +48 2250211 44, fax: +48 2250213 63, e-mail: jdomienik@tlen.pl

Received: 29.11.2020

Accepted: 17.03.2021

*Equal contribution

This article is available in open access under Creative Common Attribution-Non-Commercial-No Derivatives 4.0 International (CC BY-NC-ND 4.0) license, allowing to download articles and share them with others as long as they credit the authors and the publisher, but without permission to change them in any way or use them commercially. 


\section{Fourth universal definition of myocardial infarction: Key takeaways}

The fourth universal definition of myocardial infarction (MI) [1] introduces several changes and new concepts of MI to enhance clinical practice. The most important of them being, in the opinion of the authors herein, the distinction between MI and myocardial injury as well as an emphasis on the utility of imaging techniques - cardiovascular magnetic resonance (CMR) in defining etiology of myocardial injury and coronary computed tomography angiography in the diagnosis of MI.

The clinical definition of MI specifies: the presence of acute myocardial injury detected by abnormal cardiac biomarkers in the setting of evidence of acute myocardial ischemia [1].

In clinical practice, cardiac troponin I and troponin $\mathrm{T}$ (the latter sometimes derives from skeletal muscles [2-4]) are recommended, especially in highsensitivity cardiac troponin I (hs-cTn), mainly due to its specificity to the heart and sensitivity $[5,6]$. Of note, for the first time, the acute myocardial injury was defined clearly as detection of elevated cardiac troponin values above the $99^{\text {th }}$ percentile upper reference limit (URL) and occurrence of the rise and/or fall of focused cardiac troponin values [6]. Subsequently, without the concomitant rise and/or fall in the mentioned biomarker values, can only define chronic myocardial injury [7].

The authors emphasize the broad spectrum of clinical scenarios leading to myocardial injury, ranging from anemia, ventricular tachyarrhythmia, heart failure, kidney disease, and hypotensive shock to hypoxemia or other comorbidities (Fig. 1). However, without clinical evidence of acute ischemic myocardial injury, they should remain named "myocardial injury" in everyday practice.

For practical reasons, the authors emphasize the role of distinguishing between myocardial injury and infarction $[1,8]$. The differences are presented in Figure 2.

\section{Types of myocardial infarctions}

The types of MIs were kept, and are presented clearly in Figure 2. Type $1 \mathrm{MI}$ is defined as: the detection of a rise and/or fall of cTn with at least one value above the $99^{\text {th }}$ percentile URL and with at least one of the following:

- symptoms of acute myocardial injury;

- new ischemic electrocardiography changes;

- development of pathological Q waves;

— imaging evidence of new loss of viable myocar-

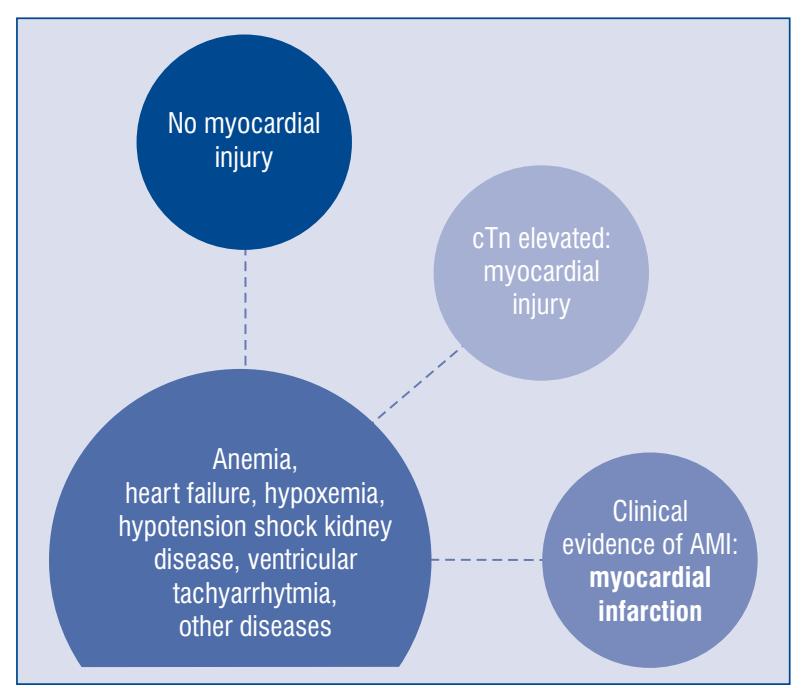

Figure 1. Spectrum of myocardial injury - from no injury to myocardial infarction; cTn - cardiac troponin; AMI - acute myocardial infarction.

dium or new regional wall motion abnormality in a pattern consistent with an ischemic etiology;

- identification of a coronary thrombus by angiography including intracoronary imaging or by autopsy [1].

The criteria for type $2 \mathrm{MI}$ do not include identification of coronary thrombus due to its mechanism - it develops secondarily to another illness or process. Possible mechanisms of imbalance between oxygen demand and oxygen supply can be fixed coronary atherosclerosis, coronary spasm, coronary embolism, coronary artery dissection, sustained tachyarrhythmia, severe bradyarrhythmia, severe hypertension, respiratory failure, shock, severe anemia or hypotension [8]. For the sake of patients, it is worth noticing that in this group, patient treatment should be based on restoration of the balance between oxygen demand and supply, through different interventions, concerning its primary cause, for instance heartrate control, blood pressure-lowering or volume adjustment $[8,9]$.

Herein, the aim is to emphasize a fundamental issue concerning this document - differences between type $1 \mathrm{MI}$, type $2 \mathrm{MI}$, and non-ischemic myocardial injury.

Type $3 \mathrm{MI}$ is very rare, constituting $3-4 \%$ of all MIs [10]. The authors highlight the difference between type $3 \mathrm{MI}$, it means death from probable cardiac reasons and sudden death from clearly non-cardiac causes (which is more 


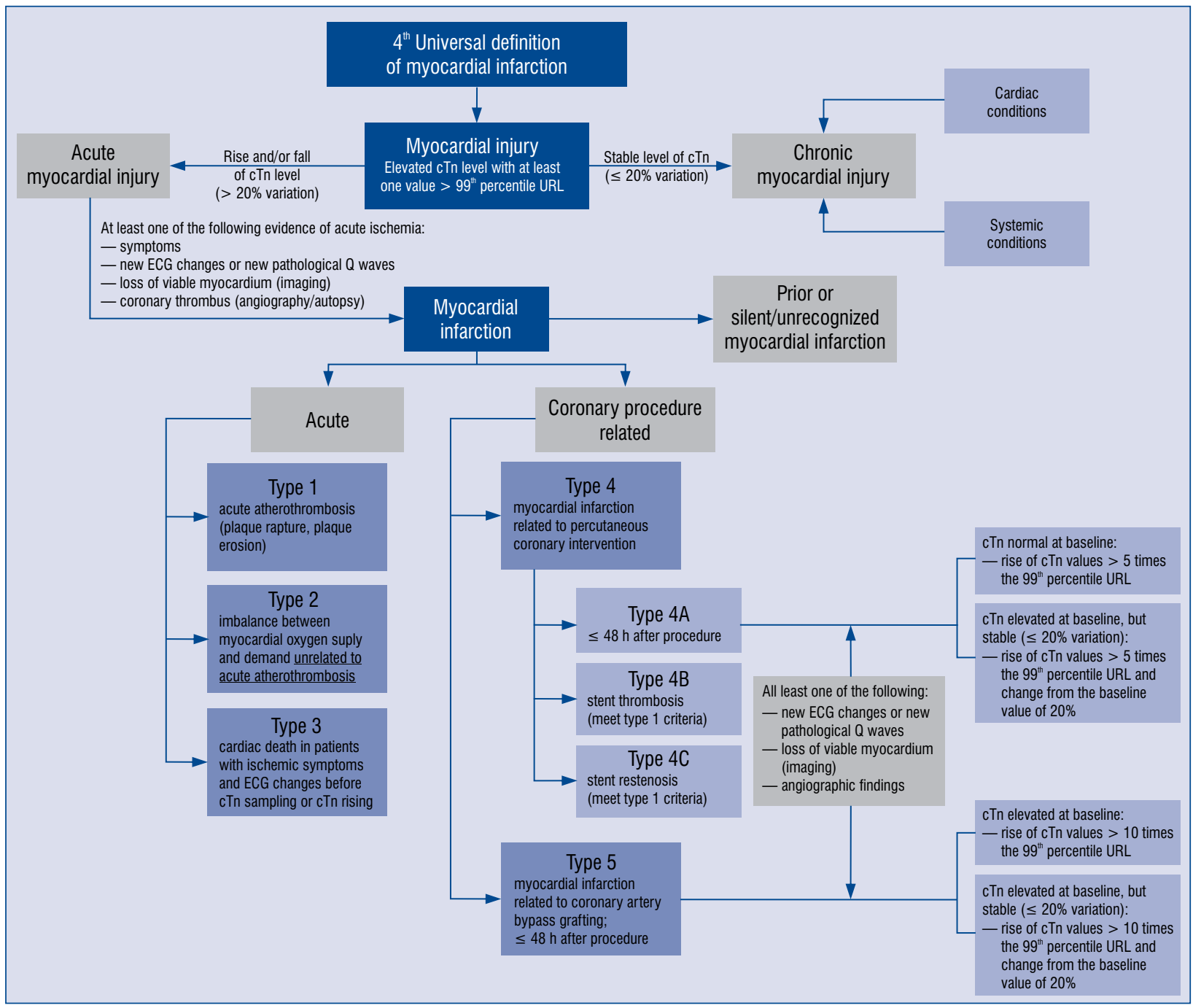

Figure 2. Scheme to distinguish myocardial injury and myocardial infarction and particular types of myocardial infarction; cTn - cardiac troponin; ECG — electrocardiogram; URL — upper reference limit.

frequent). Of note, when autopsy finds fresh or recent thrombus in the myocardial infarct-related artery, one should confirm type $1 \mathrm{MI}$ instead of type 3 MI $[11,12]$.

The document clarifies the difference between periprocedural myocardial injury and MI, both percutaneous coronary intervention (PCI) and coronary artery bypass grafting (CABG). Consequently, it emphasizes the role of cardiac biomarkers' level and stability, before further evaluations and prior to regarding as a reference level for a particular patient. The situation in which an increased level of a cardiac biomarker is potentially the result of the MI and not the procedures mentioned above [13]. The diagnosis of a periprocedural myocardial injury requires:
- increase in cardiac biomarkers (cTn) level, when initially patient presents normal values or;

— increase in cardiac biomarkers $(>20 \%)$, when initially patient shows its values above the $99^{\text {th }}$ percentile URL.

For periprocedural $(\leq 48 \mathrm{~h})$ MI related to the PCI (type 4a MI) and CABG (type $5 \mathrm{MI}$ ), five times and ten times increase in cTn value is required, respectively, if the patient presents normal initial values. These conditions are presented in Figure 3. In case of initial values of cTn above $99^{\text {th }}$ percentile URL, a $20 \%$ rise is demanded, and the final value higher than five times $99^{\text {th }}$ percentile URL or ten times $99^{\text {th }}$ percentile URL in case of CABG. They must, of course, be accompanied by one of the known clinical criteria [1]. 


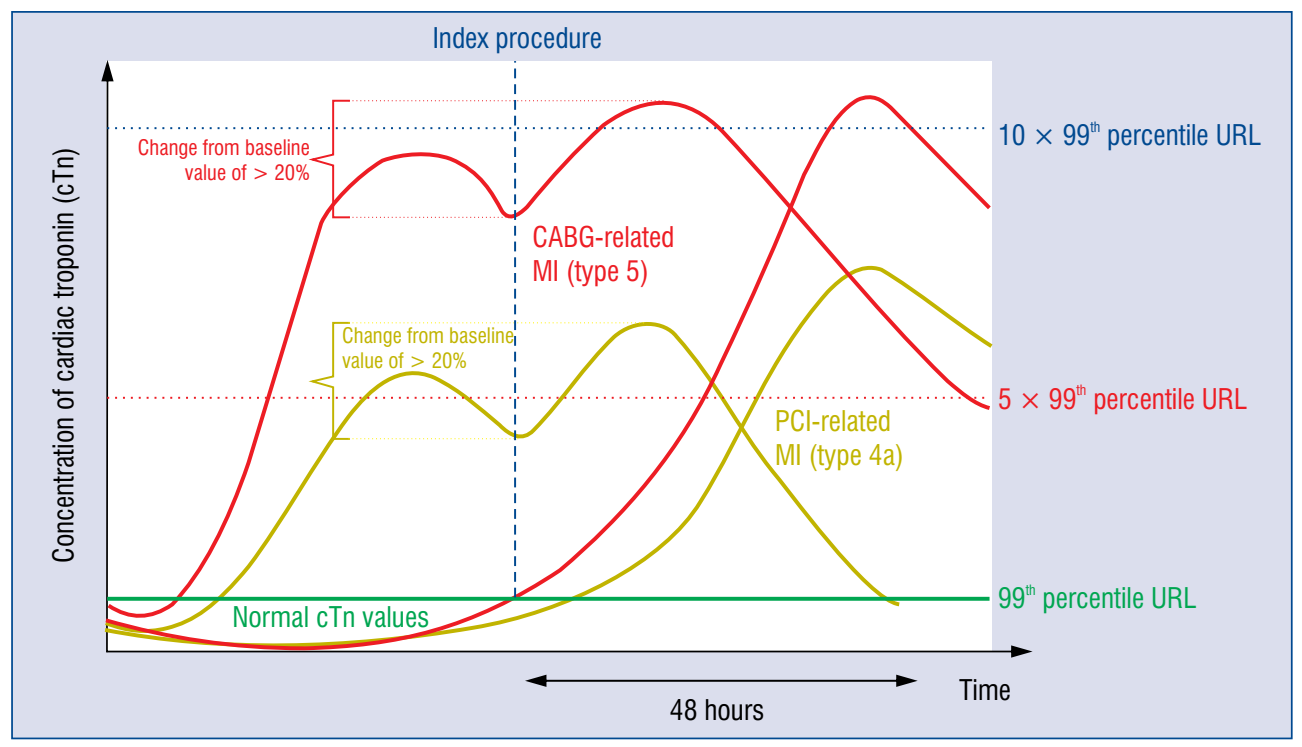

Figure 3. Concentration of cardiac troponin in different clinical scenarios relevant to revascularization procedures; cTn - cardiac troponin; CABG — coronary artery bypass grafting; PCl — percutaneous coronary intervention; URL — upper reference limit.

The definition of type 4c MI, connected with focal or diffuse restenosis after PCI, is based on a rise and/or fall of cTn values above the $99^{\text {th }}$ percentile URL and definition based on the recognition of type $1 \mathrm{MI}$ [1].

\section{Myocardial injury and infarction associated with non-cardiac procedures}

The occurrence of asymptomatic perioperative MI is strongly associated with 30-day mortality [14, 15]. Increased oxygen demand in the perioperative period and predominant etiology of myocardial ischemia are well recognized and the fact that about $35 \%$ of patients reveal hs-cTn level above the $99^{\text {th }}$ percentile URL in post-operative blood samples [16, 17]. Therefore, increased vigilance is demanded in all high-risk individuals, and their baseline pre-operative value is necessary to collect.

\section{Myocardial infarction with non-obstructive coronary arteries}

The document also highlights the diagnosis of myocardial infarction with non-obstructive coronary arteries (MINOCA; $\leq 50 \%$ diameter stenosis in a major epicardiac vessel) [1]. The prevalence of MINOCA depends on sex (it occurs more frequently in women than men), the type of MI (it is more common in non-ST-segment eleva- tion myocardial infarction [NSTEMI] than in ST-segment elevation myocardial infarction [STEMI]), and it concerns about $6-8 \%$ of patients with MI [18]. Multiple pathomechanisms underlie this condition and the heterogeneous group involves both coronary and non-coronary causes. The first authoritative international expert definition of MINOCA was published in the European Society of Cardiology working group position paper [18, 19]. Recently, the 2020 non-ST-segment elevation-acute coronary syndrome (NSTE-ACS) guidelines have maintained the approach to MINOCA as 'working diagnosis' [20] and the authors have proposed a clinical algorithm to aid in the diagnosis. The proposed 'traffic light' scheme includes different imaging tools such as echocardiography, cardiac ventriculography, CMR, intravascular imaging (intravascular ultrasonography [IVUS] or optical coherence tomography [OCT]) and intracoronary functional testing (acetylcholine or ergonovine). The most important recommendation seems to be to perform CMR in all MINOCA patients without an apparent underlying cause [20]. Patients with MINOCA can fulfil the criteria of MI type 1 and type 2 [21]. It should be stated that the current definition excludes Takotsubo syndrome (TTS) and myocarditis [20].

\section{Takotsubo syndrome}

According to available research, the authors underline for the first time, the relevance of TTS. 
They focus on the discrepancy between the usually modest and transient increases in cTn values and the large territory of electrocardiography changes or left ventricle regional akinesis or hypokinesis including apical ( $82 \%$ of patients), mid-ventricular $(14.6 \%)$, basal $(2.2 \%)$, or focal $(1.5 \%)$ territory. In TTS, the coronary arteries are usually angiographically intact while left ventriculography presents above mentioned regional wall motion abnormalities - in 10-15\% of patients [1]. On the other hand, recently published analysis from the largest InterTAK Registry concludes that coronary artery disease may coexist in TTS patients, presents with the whole spectrum of coronary pathology including acute coronary occlusion, and is associated with adverse outcome [19]. Thus, the differential diagnosis with MI can be challenging [20,21].

Consequently, unlike the previous attitude to TTS [19], the current guidelines classify it as 'other causes of myocardial injury' [1] or 'specific non-MINOCA status' [21]. However, TTS's diagnosis cannot be certainly stated in the acute phase because imaging follow-up is essential to prove the recovery of left ventricular function [21].

\section{Spontaneous coronary dissection}

Spontaneous coronary dissection (SCAD) leading to blood accumulation within the artery's false lumen with potential compression of the true lumen is an important non-atherosclerotic condition of MI [1]. It is triggered by vasa vasorum hemorrhage or intimal tear [21]. The NSTE-ACS guidelines specify three angiographic types of SCAD: type 1 with multiple radiolucent lumen, type 2 with long diffuse or smooth stenosis, and type 3 with focal or tubular stenosis [20]. The dissection coexisting with acute myocardial injury and evidence of ischemia is type 2 of MI. If coronary arteries are non-obstructive (stenosis $<50 \%$ ) the criteria of MINOCA are fulfilled [1, 21]. SCAD can be missed on coronary computed tomography angiography, therefore OCT or IVUS are applicable in unclear clinical scenarios [21].

\section{Cardiac magnetic resonance imaging}

The accuracy of CMR provides an unequivocal assessment of the etiology of myocardial injury, allowing the repeated distinction between acute vs. chronic myocardial injury. It also identifies the presence and involvement of myocardial inflammation, thus providing a clear distinction between ischemic scar/fibrosis (extending from subendo-

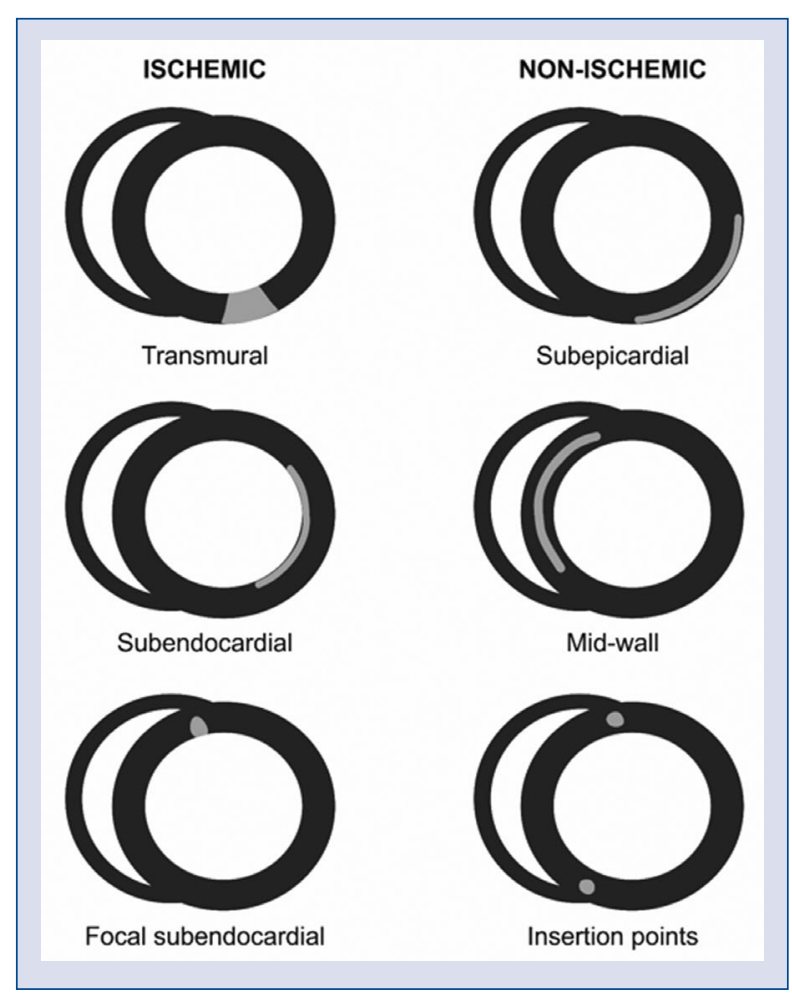

Figure 4. The different patterns of scarring in post-contrast cardiac magnetic resonance images - late-gadolinium enhancement.

cardium to endocardium) and non-ischemic scar/ /fibrosis (subepicardial, mid-wall, insertion points) in myocardial injury (Fig. 4) [1].

\section{COVID-19 and myocardial infarction}

Since the initial outbreak of the novel coronavirus disease - severe acute respiratory syndrome coronavirus 2 (SARS-CoV-2)/coronavirus disease 2019 (COVID-19) - in December 2019, data from many countries (Italy, Spain, Switzerland, The United States of America) underline a dramatic drop in the number of ACS referrals to cardiovascular centers at the time of the COVID-19 outbreak, moreover reduction of PCI in STEMI patients was $38 \%$ [22-25]. In addition, Legutko et al. [26] and Siudak et al. [27] showed a greater decline in the number of procedures for NSTEMI, unstable angina or chronic coronary syndrome than in those for STEMI (19.2\% vs. $16.2 \%)$. What is more, the decline of PCI procedures in NSTEMI after lockdown reached about 30\% [27]. Of course, the obvious consequence of this situation is the staggering growth in MI complications, such as increased morbidity and mortality. Among the 
mechanisms potentially decreasing admission to hospitals during the COVID-19 pandemic are: fear of contagion, relaxing lifestyle, decrease of air pollution, increase in pain threshold, which leads to a higher prevalence of silent or near silent MIs [28]. The need for urgent treatment according to the guidelines while maintaining the safety of medical personnel is necessary. Of note, invasive coronary angiography in acute ST-segment elevation coronary syndrome cannot be neglected, even in COVID-19 patients with myocarditis pretending to be ACS.

\section{Key points from STEMI and NSTE-ACS guidelines}

\section{STEMI}

- Some patients with coronary artery occlusion or global ischemia do not have typical ST-segment elevation in ECG. However, patients with clinical manifestation of ongoing myocardial ischemia and other ECG patterns (e.g., bundle branch block, ventricular pacing, hyperacute T-waves, isolated ST-segment depression in anterior leads, and/or universal ST-segment depression with ST-segment elevation in aVR) should be qualified for a primary PCI.

- Non-invasive imaging in STEMI patients plays a crucial role in the acute phase and during long-term management.

- The MINOCA coexisting with ST-segment elevation in ECG requires additional tests to diagnose the etiology and tailor proper management [29].

\section{NSTE-ACS}

- Myocyte injury is related to the release of troponin as intracellular protein into the systemic circulation and elevated troponin level is a marker of myocardial injury, not only a marker of MI. Troponin results should be interpreted in the clinical context.

- Patients with MINOCA can fulfil the criteria of MI type 1 and type 2 [21]. The 2020 NSTE-ACS guidelines have proposed a clinical algorithm to aid in the diagnosis of MINOCA, including different imaging tools such as echocardiography, cardiac ventriculography, CMR, intravascular imaging (IVUS or OCT) and intracoronary functional testing (acetylcholine or ergonovine). The most important recommendation seems to be to perform CMR in all MINOCA patients without an apparent underlying cause [20].
- hs-cTn assays have a higher negative predictive value for acute MI than standard troponin tests.

- Higher sensitivity and diagnostic accuracy for the diagnosis of MI enables shortening the time interval between the first and second hs-cTn assessment. The $0 \mathrm{~h} / 1 \mathrm{~h}$ rule-in or rule-out algorithm first and $0 \mathrm{~h} / 2 \mathrm{~h}$ second should be chosen. The cut-off values within both protocols are assay-specific and baseline level, acute change must be taken into account $(1 \mathrm{~h} \Delta$ or $2 \mathrm{~h} \Delta)$. Additional blood draw after $3 \mathrm{~h}$ should be done if previous troponin assessment $(0 \mathrm{~h} / 1 \mathrm{~h})$ is inconclusive and clinical status still suggests ACS. A rule-out $0 \mathrm{~h} / 3 \mathrm{~h}$ protocol is still recommended but with the lower level of recommendation [20].

\section{Conflict of interest: None declared}

\section{References}

1. Thygesen K. 'Ten Commandments' for the Fourth Universal Definition of Myocardial Infarction 2018. Eur Heart J. 2019; 40(3): 226, doi: 10.1093/eurheartj/ehy856, indexed in Pubmed: 30649367.

2. Rittoo D, Jones A, Lecky B, et al. Elevation of cardiac troponin T, but not cardiac troponin I, in patients with neuromuscular diseases: implications for the diagnosis of myocardial infarction. J Am Coll Cardiol. 2014; 63(22): 2411-2420, doi: 10.1016/j. jacc.2014.03.027, indexed in Pubmed: 24747102.

3. Jaffe AS, Vasile VC, Milone M, et al. Diseased skeletal muscle: a noncardiac source of increased circulating concentrations of cardiac troponin T. J Am Coll Cardiol. 2011; 58(17): 1819-1824, doi: 10.1016/j.jacc.2011.08.026, indexed in Pubmed: 21962825.

4. Wens SCA, Schaaf GJ, Michels M, et al. Elevated plasma cardiac troponin $\mathrm{T}$ levels caused by skeletal muscle damage in pompe disease. Circ Cardiovasc Genet. 2016; 9(1): 6-13, doi: 10.1161/ CIRCGENETICS.115.001322, indexed in Pubmed: 26787432.

5. Thygesen K, Mair J, Katus H, et al. Recommendations for the use of cardiac troponin measurement in acute cardiac care. Eur Heart J. 2010; 31(18): 2197-2204, doi: 10.1093/eurheartj/ehq251, indexed in Pubmed: 20685679.

6. Thygesen K, Mair J, Giannitsis E, et al. How to use high-sensitivity cardiac troponins in acute cardiac care. Eur Heart J. 2012; 33(18): 2252-2257, doi: 10.1093/eurheartj/ehs154, indexed in Pubmed: 22723599.

7. Sarkisian L, Saaby L, Poulsen T, et al. Prognostic impact of myocardial injury related to various cardiac and noncardiac conditions. Am J Med. 2016; 129(5): 506-514.e1, doi: 10.1016/j. amjmed.2015.12.009.

8. Januzzi JL, Sandoval Y. The many faces of type 2 myocardial infarction. J Am Coll Cardiol. 2017; 70(13): 1569-1572, doi: 10.1016/j.jacc.2017.07.784, indexed in Pubmed: 28935033.

9. Roffi M, Patrono C, Collet JP, et al. 2015 ESC Guidelines for the management of acute coronary syndromes in patients presenting without persistent ST-segment elevation. Eur Heart J. 2015; 37(3): 267-315, doi: 10.1093/eurhearti/ehv320.

10. Jangaard N, Sarkisian L, Saaby L, et al. Incidence, frequency, and clinical characteristics of type 3 myocardial infarction in clinical 
practice. Am J Med. 2017; 130(7): 862.e9-862.e14, doi: 10.1016/j. amjmed.2016.12.034, indexed in Pubmed: 28159605.

11. Thygesen K, Alpert JS, White HD. Universal definition of myocardial infarction. Eur Heart J. 2007; 28(20): 2525-2538, doi: 10.1093/eurheartj/ehm355, indexed in Pubmed: 17951287.

12. Thygesen K, Alpert JS, Jaffe AS, et al. Third universal definition of myocardial infarction. Eur Heart J. 2012; 33(20): 2551-2567, doi: 10.1093/eurheartj/ehs184, indexed in Pubmed: 22922414.

13. Rahimi K, Banning AP, Cheng ASH, et al. Prognostic value of coronary revascularisation-related myocardial injury: a cardiac magnetic resonance imaging study. Heart. 2009; 95(23): 1937-1943, doi: 10.1136/hrt.2009.173302, indexed in Pubmed: 19687014.

14. Devereaux PJ, Xavier D, Pogue J, et al. Characteristics and short-term prognosis of perioperative myocardial infarction in patients undergoing noncardiac surgery: a cohort study. Ann Intern Med. 2011; 154(8): 523-528, doi: 10.7326/0003-4819-1548-201104190-00003, indexed in Pubmed: 21502650.

15. Devereaux PJ, Chan MTV, Alonso-Coello P, et al. Association between postoperative troponin levels and 30-day mortality among patients undergoing noncardiac surgery. JAMA. 2012; 307(21): 22952304, doi: 10.1001/jama.2012.5502, indexed in Pubmed: 22706835.

16. Kavsak PA, Walsh M, Srinathan S, et al. High sensitivity troponin $\mathrm{T}$ concentrations in patients undergoing noncardiac surgery: a prospective cohort study. Clin Biochem. 2011; 44(12): 1021-1024, doi: 10.1016/j.clinbiochem.2011.05.017, indexed in Pubmed: 21640092.

17. Duvall WL, Sealove B, Pungoti C, et al. Angiographic investigation of the pathophysiology of perioperative myocardial infarction. Catheter Cardiovasc Interv. 2012; 80(5): 768-776, doi: 10.1002/ccd.23446, indexed in Pubmed: 22419582.

18. Agewall S, Beltrame JF, Reynolds HR, et al. ESC working group position paper on myocardial infarction with non-obstructive coronary arteries. Eur Heart J. 2017; 38(3): 143-153, doi: 10.1093/ eurheartj/ehw149, indexed in Pubmed: 28158518.

19. Napp LC, Cammann VL, Jaguszewski M, et al. Coexistence and outcome of coronary artery disease in Takotsubo syndrome. Eur Heart J. 2020; 41(34): 3255-3268, doi: 10.1093/eurheartj/ ehaa210, indexed in Pubmed: 32484517.

20. Templin C, Ghadri JR, Diekmann J, et al. Clinical features and outcomes of takotsubo (stress) cardiomyopathy. N Engl J Med. 2015; 373(10): 929-938, doi: 10.1056/NEJMoa1406761, indexed in Pubmed: 26332547.
21. Collet JP, Thiele H. Lesson learnt from the new 2020 ESC guidelines on non-ST-segment elevation acute coronary syndrome: when clinical judgement precedes and overpasses weak recommendations. 2020 non-ST-segment elevation acute coronary syndrome guidelines on pre-treatment: primum non nocere! Eur Heart J. 2020 [Epub ahead of print], doi: 10.1093/eurheartj/ ehaa861, indexed in Pubmed: 33205199.

22. Garcia S, Albaghdadi MS, Meraj PM, et al. Reduction in ST-segment elevation cardiac catheterization laboratory activations in the United States during COVID-19 pandemic. J Am Coll Cardiol. 2020; 75(22): 2871-2872, doi: 10.1016/j. jacc.2020.04.011, indexed in Pubmed: 32283124.

23. Rodriguez-Leor O, Cid-Alvarez B. STEMI care during COVID-19: losing sight of the forest for the trees. JACC Case Rep. 2020; 2(10): 1625-1627, doi: 10.1016/j.jaccas.2020.04.011.

24. Holy EW, Jakob P, Manka R, et al. Impact of a nationwide COVID-19 lockdown on acute coronary syndrome referrals. Cardiol J. 2020; 27(5): 633-635, doi: 10.5603/CJ.a2020.0091, indexed in Pubmed: 32643140 .

25. Wańha W, Wybraniec M, Kapłon-Cieślicka A, et al. Myocardial infarction in the shadow of COVID-19. Cardiol J. 2020; 27(5): 478-480, doi: 10.5603/CJ.2020.0152, indexed in Pubmed: 33165896.

26. Legutko J, Niewiara $€$, Bartuś S, et al. Decline in the number of coronary angiography and percutaneous coronary intervention procedures in patients with acute myocardial infarction in Poland during the coronavirus disease 2019 pandemic. Kardiol Pol. 2020; 78(6): 574-576, doi: 10.33963/KP.15393, indexed in Pubmed: 32469190.

27. Siudak $Z$, Grygier M, Wojakowski W, et al. Clinical and procedural characteristics of COVID-19 patients treated with percutaneous coronary interventions. Catheter Cardiovasc Interv. 2020; 96(6): E568-E575, doi: 10.1002/ccd.29134, indexed in Pubmed: 32686899.

28. Niccoli G, Luescher TF, Crea F. Decreased myocardial infarction admissions during COVID times: what can we learn? Cardiovasc Res. 2020; 116(10): e126-e128, doi: 10.1093/cvr/cvaa146, indexed in Pubmed: 32463078.

29. Ibanez B, James S, Agewall S, et al. [2017 ESC Guidelines for the management of acute myocardial infarction in patients presenting with ST-segment elevation.]. Kardiol Pol. 2018; 76(2): 229-313, doi: 10.5603/KP.2018.0041, indexed in Pubmed: 29457615. 